\title{
43. DATA REPORT: MINERALOGY OF SITE 884 ${ }^{1}$
}

\author{
Eve Arnold ${ }^{2}$
}

\section{INTRODUCTION}

Hole $884 \mathrm{~B}\left(51^{\circ} 27^{\prime} \mathrm{N}, 168^{\circ} 20^{\prime} \mathrm{E}\right)$ was drilled at about $3800 \mathrm{~m}$ water depth in the Meiji Drift deposit of the North Pacific. A total of $854 \mathrm{~m}$ of sediments were recovered at Hole 884B. Two stratigraphic sedimentary units were recognized at this site: a Quaternary to early Miocene clay and clayey diatom ooze unit, and an early Miocene to early Eocene claystone, chalk, and conglomerate unit. This study reports the mineralogy of Unit I, which contains three subunits. Subunit IA (0-128 mbsf, Quaternary to late Pliocene) is composed of clay and diatom clay with dropstones, ash, and diatom ooze as minor lithologies. Subunit IB (128-440 mbsf, late Pliocene to late Miocene) is clayey diatom ooze, with smaller amounts of ash and no dropstones. Subunit IC (440-546 mbsf, late Miocene to middle Miocene) is claystone, with no ash layers.

\section{METHODS}

Samples for mineralogy analyses were collected at approximately $10 \mathrm{~m}$ intervals, with one $10 \mathrm{~cm}^{3}$ sample per core. Samples were freeze-dried and weighed, then wet sieved at $63 \mu \mathrm{m}$; the $>63 \mu \mathrm{m}$ and $<63 \mu \mathrm{m}$ fractions were dried and weighed. The $<63 \mu \mathrm{m}$ fraction was treated with buffered acetic acid to remove calcium carbonate; biogenic silica was removed using a sodium carbonate procedure and iron oxides were removed using the oxalic acid extraction technique of Landa and Gast (1973). Sediments were saturated with $\mathrm{MgCl}_{2}$ to reduce $\mathrm{d}$-spacing variability caused by cation differences; they were then rinsed with warm deionized water, dried, and weighed. Sediments were wet sieved at $20 \mu \mathrm{m}$ with the aid of a sonic dismembrator, and the $<20 \mu \mathrm{m}$ fraction was split into $2-20 \mu \mathrm{m}$ and $<2 \mu \mathrm{m}$ size fractions by means of centrifugation. The $2-20 \mu \mathrm{m}$ size fraction was spiked with a $10 \%$ by weight $\mathrm{Al}_{2} \mathrm{O}_{3}$ internal standard, and the $<2 \mu \mathrm{m}$ fraction was spiked with a $10 \%$ talc internal standard. Samples were homogenized by grinding in a mortar and pestle under acetone; they were then air dried and packed into plastic filter holders for X-ray analysis. The $\mathrm{X}$-ray analysis was run from $2^{\circ}$ to $30^{\circ} 2 \theta$ at $45 \mathrm{kV}$ and $40 \mathrm{~mA}$ at $2^{\circ} 2 \theta / \mathrm{min}$ using $\mathrm{CuK} \alpha$ radiation. Peak areas for smectite, illite, amphibole, kaolinite, chlorite, quartz, and plagioclase were determined using Scintag DMS software. The relative proportion of kaolinite and chlorite were determined by the relative proportions of the kaolinite [002] and chlorite [004] peak areas. Mineral peak areas were normalized to the internal standard peak areas. Normalized peak areas were converted to weight percent using the corrected Biscaye weighting factors given in Heath and Pisias (1979).

\section{RESULTS}

Figures 1 and 2 illustrate the $<2 \mu \mathrm{m}$ and $2-20 \mu \mathrm{m}$ wt $\%$ of smectite, illite, chlorite, quartz, and plagioclase of sediments recovered from

\footnotetext{
'Rea, D.K., Basov, I.A., Scholl, D.W., and Allan, J.F. (Eds.), 1995. Proc. ODP, Sci. Results, 145: College Station, TX (Ocean Drilling Program).

${ }^{2}$ University of Rhode Island, Graduate School of Oceanography, Narragansett, RI 02882, U.S.A.
}

Hole 884B. There were only a few examples of sediments containing kaolinite or amphibole; these minerals are not discussed, therefore. For the $<2 \mu \mathrm{m}$ fraction, smectite, illite, chlorite, quartz, and plagioclase show the highest weight percent in Subunit IA, with a concentration peak at 70-90 mbsf; Subunits $1 \mathrm{~B}$ and $1 \mathrm{C}$ demonstrate little compositional variability. The $<2 \mu \mathrm{m}$ mineralogy is presented in Table 1 . The $2-20 \mu \mathrm{m}$ size fraction is more variable than the $<2 \mu \mathrm{m}$ size fraction, with concentration peaks in all mineral groups centered at 20 and 100 $\mathrm{mbsf}$. There is some suggestion of a concentration increase in smectite, quartz, and plagioclase from about $400 \mathrm{mbsf}$ toward the bottom of Subunit IC. The $2-20 \mu \mathrm{m}$ mineralogy is presented in Table 2 .

Figures 3 and 4 illustrate mineral ratios and the percent diffracting mineralogy for the $<2 \mu \mathrm{m}$ and $2-20 \mu \mathrm{m}$ size fractions. With the exception of the $2-20 \mu \mathrm{m}$ chlorite/illite and smectite/illite, the mineral ratios indicate a very uniform mineralogy for the Unit I sediments of Hole 884B. There is a peak in the chlorite/illite and smectite/illite ratios in the $2-20 \mu \mathrm{m}$ size fraction from about 225 to $250 \mathrm{mbsf}$. The smectite/ illite ratio is low in the top of Subunits IB and IA above this interval.

The percent diffracting mineralogy represents the sum of the wellcrystallized phases present in the sediment. Nondiffracting material includes amorphous silica, organic matter, and poorly crystallized mineral particles. The $<2 \mu \mathrm{m}$ size fraction shows a small increase from the bottom to the top of Unit I in the percent diffracting mineralogy, with a peak centered at $70-90 \mathrm{mbsf}$. The $2-20 \mu \mathrm{m} \%$ diffracting mineralogy is more variable than the $<2 \mu \mathrm{m}$ size fraction, with Subunit IB yielding the lowest concentration of diffracting material.

\section{SUMMARY}

The mineralogy of sediments recovered at Hole $884 \mathrm{~B}$ is remarkably uniform. Comparison of the mineral weight percent (Figs. 1 and 2) with the percent diffracting mineralogy (Figs. 3 and 4) for each size class shows that each mineral group covaries with the percent diffracting material in the same manner. This indicates that the variation in the concentration of individual mineral groups is almost completely explained by dilution with amorphous, or nondiffracting, material. The diffraction patterns indicated that not all of the amorphous silica (in this case, diatoms) was removed by the sodium carbonate extraction as indicated by a broad increase in background starting at about $20^{\circ} 2 \theta$.

The constant mineral peak ratios (illustrated in Figs. 3 and 4) indicate that the mineralogy of Unit $\mathrm{I}$ is homogeneous. The exception to this monotony is the interval from 225 to $250 \mathrm{mbsf}$ in the $2-20 \mu \mathrm{m}$ size fraction, where there is a three- to fourfold increase in the smectite/ illite and chlorite/illite ratio. The cause of this anomaly may be diagenetic, as there is no notable change in the lithology indicated in the core descriptions (Rea, Basov, Janecek, Palmer-Julson, et al., 1993). However, as no clear evidence is present for diagenetic alteration in this interval based on the geochemistry generated aboard the JOIDES Resolution (Rea, Basov, Janecek, Palmer-Julson, et al., 1993), this change in mineralogy remains enigmatic.

\section{ACKNOWLEDGMENTS}

Eve Arnold was supported by a JOI fellowship for this research. This study benefited from the kind assistance of the ODP staff and SEDCO 

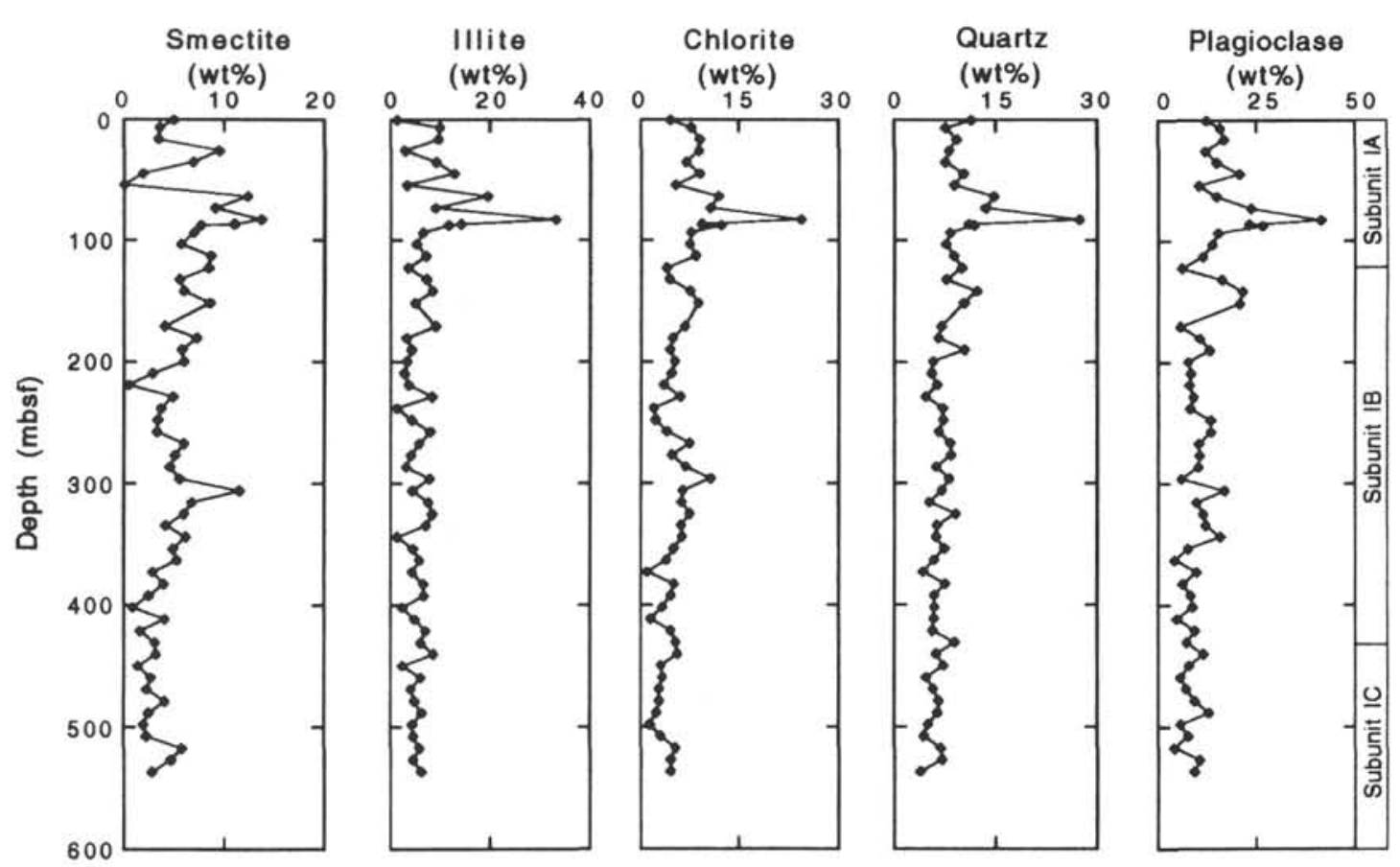

Figure 1. Mineral weight percent vs. depth for the $<2 \mu \mathrm{m}$ size fraction.
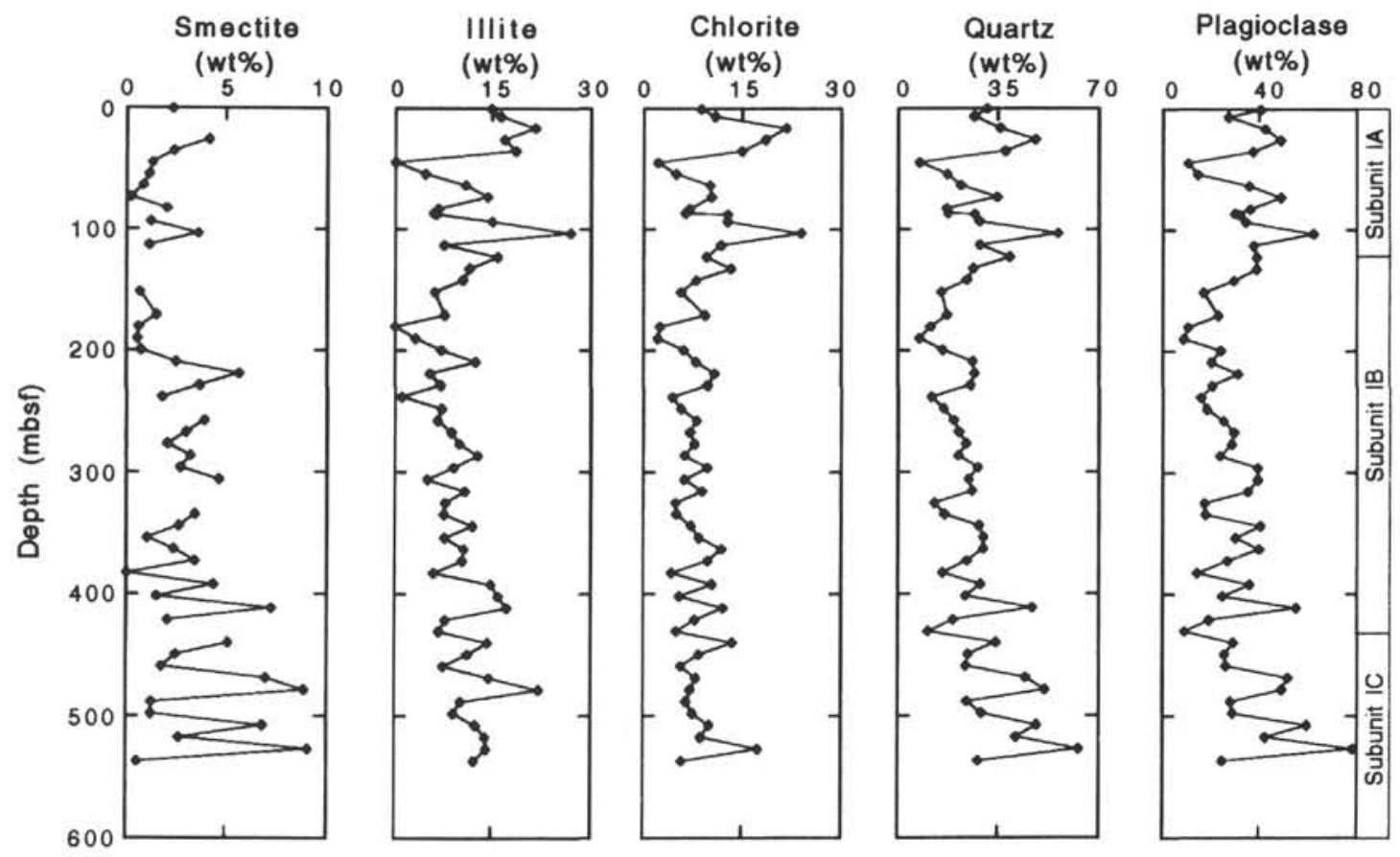

Figure 2. Mineral weight percent vs. depth for the $2-20 \mu \mathrm{m}$ size fraction.

crew aboard the JOIDES Resolution. Ms. Bonnie Wolfe assisted in the processing of the sediments. Thomas $\mathrm{C}$. Johnson reviewed the manuscript. I thank these individuals for their contributions.

\section{REFERENCES}

Heath, G.R., and Pisias, N.G., 1979. A method for the quantitative estimation of clay minerals in North Pacific deep-sea sediments. Clays Clay Miner., $27: 175-184$.

Landa, E.R., and Gast, R.G., 1973. Evaluation of crystallinity in hydrated ferric oxides. Clays Clay Miner., 21:121-130.
Rea, D.K., Basov, I.A., Janecek, T.R., Palmer-Julson, A., et al., 1993. Proc. ODP, Init. Repts., 145: College Station, TX (Ocean Drilling Program). Abbreviations for names of organizations and publications in ODP reference lists follow
the style given in Chemical Abstracts Service Source Index (published by American
Chemical Society).

Date of initial receipt: 9 May 1994

Date of acceptance: 1 August 1994

Ms 145SR-142 

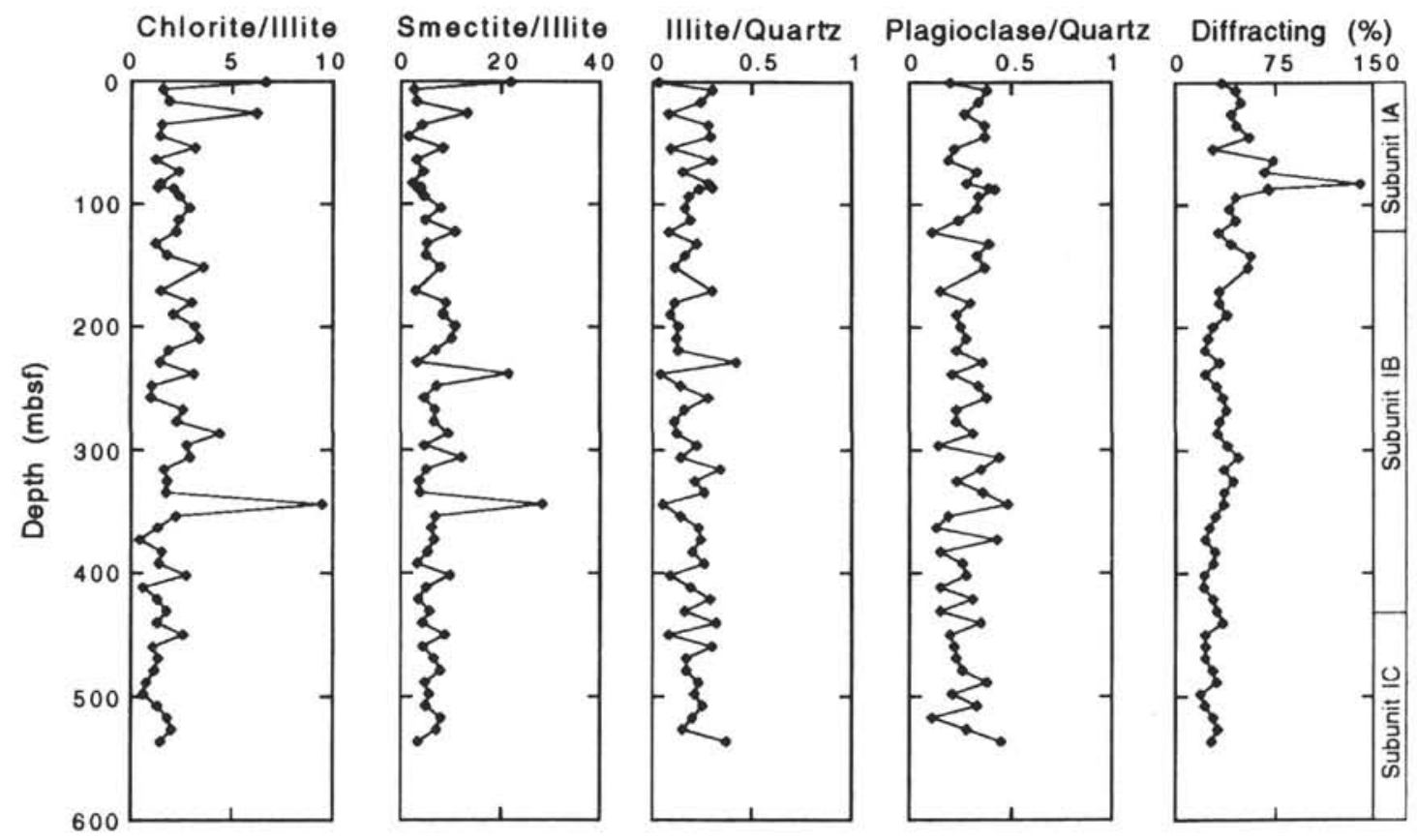

Figure 3. Mineral peak area ratios and percent diffracting mineralogy for the $<2 \mu \mathrm{m}$ size fraction.
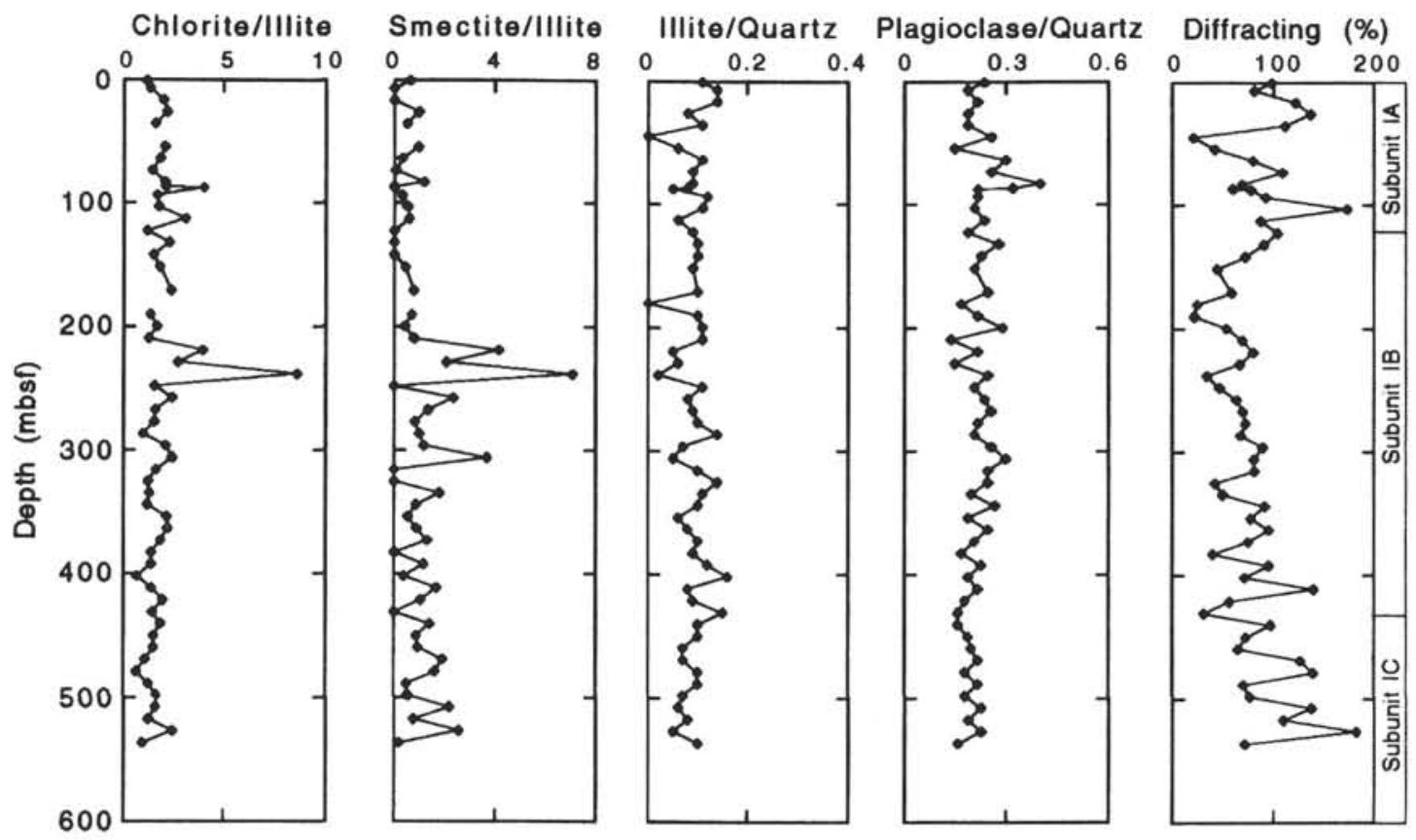

Figure 4. Mineral peak area ratios and percent diffracting mineralogy for the 2-20 $\mu \mathrm{m}$ size fraction. 
Table 1. Mineral weight percent for the $<2 \mu \mathrm{m}$ size fraction, Hole $884 \mathrm{~B}$.

\begin{tabular}{|c|c|c|c|c|c|c|}
\hline $\begin{array}{l}\text { Core, section, } \\
\text { interval }(\mathrm{cm})\end{array}$ & $\begin{array}{l}\text { Depth } \\
\text { (mbsf) }\end{array}$ & $\begin{array}{l}\text { Smectite } \\
\text { (wt\%) }\end{array}$ & $\begin{array}{l}\text { Illite } \\
\text { (wt\%) }\end{array}$ & $\begin{array}{l}\text { Chlorite } \\
\text { (wt\%) }\end{array}$ & $\begin{array}{l}\text { Quartz } \\
\text { (wt\%) }\end{array}$ & $\begin{array}{l}\text { Plagioclase } \\
\text { (wt\%) }\end{array}$ \\
\hline \multicolumn{7}{|l|}{$145-884 \mathrm{~B}-$} \\
\hline $1 \mathrm{H}-1,3 \mathrm{I}-33$ & 0.31 & 5.00 & 1.38 & 4.59 & 11.53 & 12.31 \\
\hline $2 \mathrm{H}-1,30-32$ & 6.80 & 3.60 & 10.00 & 7.84 & 7.71 & 15.75 \\
\hline $3 \mathrm{H}-1,30-32$ & 16.30 & 3.50 & 9.75 & 9.20 & 9.36 & 16.75 \\
\hline $4 \mathrm{H}-1,30-32$ & 25.80 & 9.50 & 2.89 & 9.00 & 8.13 & 11.87 \\
\hline $5 \mathrm{H}-1,30-32$ & 35.30 & 6.90 & 9.15 & 6.87 & 7.56 & 14.80 \\
\hline $6 \mathrm{H}-1,30-32$ & 44.80 & 1.90 & 12.88 & 9.12 & 10.32 & 20.54 \\
\hline $7 \mathrm{H}-1,31-33$ & 54.31 & 0.00 & 3.33 & 5.27 & 8.92 & 10.27 \\
\hline $8 \mathrm{H}-1,3 \mathrm{l}-33$ & 63.81 & 12.30 & 19.51 & 11.94 & 14.77 & 14.80 \\
\hline $9 \mathrm{H}-1,31-33$ & 73.31 & 9.10 & 9.10 & 10.78 & 13.54 & 23.56 \\
\hline $10 \mathrm{H}-1,30-32$ & 82.80 & 13.70 & 33.11 & 24.32 & 27.36 & 41.32 \\
\hline $11 \mathrm{H}-1,27-29$ & 86.57 & 11.00 & 14.29 & 9.41 & 11.05 & 23.06 \\
\hline $12 \times-1,30-32$ & 87.60 & 7.70 & 11.74 & 12.36 & 11.80 & 26.47 \\
\hline $13 X-1,36-38$ & 93.66 & 7.00 & 6.48 & 7.71 & 8.29 & 15.16 \\
\hline $14 X-1,33-35$ & 103.33 & 5.70 & 5.22 & 7.55 & 7.71 & 13.71 \\
\hline $15 X-1,31-33$ & 112.91 & 8.70 & 7.23 & 8.49 & 8.93 & 11.32 \\
\hline $16 X-1,30-32$ & 122.50 & 8.50 & 3.58 & 3.98 & 10.03 & 5.90 \\
\hline $17 X-1,31-33$ & 132.11 & 5.60 & 7.30 & 4.41 & 7.73 & 16.14 \\
\hline $18 \mathrm{X}-1,31-33$ & 141.81 & 6.00 & 8.52 & 7.61 & 12.25 & 21.44 \\
\hline $19 \mathrm{X}-1,30-32$ & 151.40 & 8.60 & 5.00 & 8.93 & 10.36 & 20.65 \\
\hline $21 \times-1,30-32$ & 170.80 & 4.10 & 9.17 & 6.75 & 7.00 & 5.64 \\
\hline $22 \mathrm{X}-1,31-33$ & 180.41 & 7.30 & 3.26 & 4.87 & 6.57 & 10.64 \\
\hline $23 X-1,30-32$ & 190.10 & 5.80 & 4.29 & 4.49 & 10.50 & 13.11 \\
\hline $24 X-1,32-34$ & 199.72 & 6.00 & 3.30 & 5.22 & 5.76 & 7.62 \\
\hline $25 X-1,32-34$ & 209.42 & 2.90 & 2.80 & 4.72 & 5.54 & 8.34 \\
\hline $26 \mathrm{X}-1,31-33$ & 219.01 & 0.50 & 3.73 & 3.50 & 6.43 & 7.99 \\
\hline $27 \mathrm{X}-1,31-33$ & 228.71 & 4.90 & 8.47 & 6.05 & 4.64 & 8.96 \\
\hline $28 X-1,30-32$ & 238.30 & 3.70 & 1.27 & 1.97 & 7.20 & 8.11 \\
\hline $29 \mathrm{X}-1,31-33$ & 247.91 & 3.40 & 4.33 & 2.23 & 7.29 & 13.33 \\
\hline $30 X-1,31-33$ & 257.61 & 3.30 & 8.08 & 4.00 & 6.61 & 13.42 \\
\hline $31 X-1,30-32$ & 267.30 & 6.00 & 5.81 & 7.50 & 8.35 & 10.29 \\
\hline $32 X-1,30-32$ & 277.00 & 5.10 & 4.18 & 4.75 & 8.47 & 10.55 \\
\hline $33 X-1,30-32$ & 286.60 & 4.60 & 3.15 & 6.89 & 6.20 & 10.16 \\
\hline $34 X-1,32-34$ & 296.32 & 5.60 & 7.92 & 10.83 & 8.16 & 5.91 \\
\hline $35 \mathrm{X}-1,31-33$ & 306.01 & 11.50 & 4.47 & 6.46 & 7.10 & 16.76 \\
\hline $36 X-1,31-33$ & 315.61 & 6.80 & 7.71 & 6.25 & 5.25 & 9.72 \\
\hline $37 X-1,30-32$ & 325.30 & 6.00 & 8.49 & 7.54 & 9.13 & 11.42 \\
\hline $38 X-1,30-32$ & 334.60 & 4.20 & 7.14 & 6.15 & 6.34 & 12.07 \\
\hline $39 \mathrm{X}-1,31-33$ & 344.21 & 6.20 & 1.33 & 6.33 & 6.17 & 15.66 \\
\hline $40 X-1,31-33$ & 353.91 & 4.90 & 4.56 & 5.05 & 7.44 & 7.54 \\
\hline $41 X-1,31-33$ & 363.41 & 5.30 & 5.90 & 3.88 & 5.88 & 4.20 \\
\hline $42 X-1,30-32$ & 373,00 & 2.90 & 4.33 & 0.91 & 4.22 & 9.75 \\
\hline $43 \mathrm{X}-1,30-32$ & 382.70 & 4.00 & 6.68 & 5.08 & 7.57 & 6.13 \\
\hline $44 X-1,30-32$ & 392.30 & 2.50 & 6.75 & 4.62 & 5.91 & 8.32 \\
\hline $45 X-1,30-32$ & 402.00 & 0.90 & 2.39 & 3.27 & 5.94 & 8.75 \\
\hline $46 \mathrm{X}-1,30-32$ & 411.60 & 4.10 & 4.89 & 1.46 & 5.88 & 4.69 \\
\hline $47 X-1,30-32$ & 421.20 & 1.60 & 7.04 & 4.59 & 5.64 & 9.23 \\
\hline $48 X-1,30-32$ & 430.90 & 3.10 & 6.09 & 5.36 & 8.97 & 7.26 \\
\hline $49 \mathrm{X}-1,30-32$ & 440.50 & 3.20 & 8.75 & 5.62 & 6.20 & 11.46 \\
\hline $50 X-1,30-32$ & 450.10 & 1.40 & 2.40 & 3.10 & 7.34 & 7.79 \\
\hline $51 X-1,30-32$ & 459.60 & 2.70 & 6.12 & 3.31 & 4.72 & 5.61 \\
\hline $52 \times-1,30-32$ & 469.20 & 2.30 & 4.14 & 2.79 & 5.77 & 7.11 \\
\hline $53 X-1,32-34$ & 478.92 & 4.10 & 4.88 & 2.81 & 6.60 & 9.30 \\
\hline $54 \mathrm{X}-1,30-32$ & 488.50 & 2.50 & 6.36 & 2.37 & 6.44 & 12.87 \\
\hline $55 \mathrm{X}-1,30-32$ & 498.10 & 2.00 & 4.46 & 1.38 & 5.00 & 5.66 \\
\hline $56 \mathrm{X}-1,30-32$ & 507.70 & 2.30 & 4.67 & 3.05 & 4.37 & 7.68 \\
\hline $57 X-1,30-32$ & 517.40 & 5.80 & 5.90 & 5.30 & 6.92 & 4.01 \\
\hline $58 \mathrm{X}-1,30-32$ & 527.00 & 4.70 & 4.52 & 4.55 & 7.08 & 10.52 \\
\hline \multirow{3}{*}{$59 \mathrm{X}-1,30-32$} & 536.70 & 2.80 & 6.24 & 4.55 & 3.83 & 9.15 \\
\hline & Average & 5.09 & 6.66 & 6.02 & 7.96 & 11.94 \\
\hline & SD & 2.93 & 4.83 & 3.60 & 3.47 & 6.41 \\
\hline
\end{tabular}

Note: $\mathrm{SD}=$ standard deviation, blank = no measurement.
Table 2. Mineral weight percent for the $\mathbf{2 - 2 0} \mu \mathrm{m}$ size fraction, Hole 884B.

\begin{tabular}{|c|c|c|c|c|c|c|}
\hline $\begin{array}{l}\text { Core, section, } \\
\text { interval }(\mathrm{cm})\end{array}$ & $\begin{array}{l}\text { Depth } \\
\text { (mbsf) }\end{array}$ & $\begin{array}{l}\text { Smectite } \\
\text { (wt\%) }\end{array}$ & $\begin{array}{l}\text { Illite } \\
(w 1 \%)\end{array}$ & $\begin{array}{l}\text { Chlorite } \\
\text { (wt\%) }\end{array}$ & $\begin{array}{l}\text { Quartz } \\
\text { (wt\%) }\end{array}$ & $\begin{array}{l}\text { Plagioclase } \\
\quad \text { (wt\%) }\end{array}$ \\
\hline \multicolumn{7}{|l|}{ 145-884B- } \\
\hline IH-I, 31-33 & 0.31 & 2.30 & 14.91 & 8.74 & 31.37 & 40.57 \\
\hline $2 \mathrm{H}-1,30-32$ & 6.80 & & 16.30 & 10.90 & 26.85 & 27.11 \\
\hline $3 \mathrm{H}-1,30-32$ & 16.30 & & 21.55 & 21.77 & 35.92 & 42.58 \\
\hline $4 \mathrm{H}-1,30-32$ & 25.80 & 4.15 & 16.89 & 18.61 & 48.07 & 48.91 \\
\hline $5 \mathrm{H}-1,30-32$ & 35.30 & 2.38 & \multirow[t]{2}{*}{18.64} & 14.95 & 37.60 & 37.45 \\
\hline $6 \mathrm{H}-1,30-32$ & 44.80 & 1.29 & & 2.17 & 7.43 & 10.32 \\
\hline $7 \mathrm{H}-1,31-33$ & 54.31 & 1.12 & 4.69 & 4.90 & 17.31 & 14.21 \\
\hline $8 \mathrm{H}-1,3 \mathrm{I}-33$ & 63.81 & 0.83 & 10.9 & 10.08 & 22.21 & 35.79 \\
\hline $9 \mathrm{H}-1,31-33$ & 73.31 & 0.22 & 14.32 & 10.36 & 34.71 & 48.94 \\
\hline $10 \mathrm{H}-1,30-32$ & 82.80 & 2.01 & 6.75 & 7.03 & 17.07 & 36.23 \\
\hline $11 \mathrm{H}-1,27-29$ & 86.57 & & 6.00 & 6.38 & 17.67 & 29.81 \\
\hline $12 X-1,30-32$ & 87.60 & & 6.37 & 12.83 & 27.07 & 31.79 \\
\hline $13 \mathrm{X}-1,36-38$ & 93.66 & 1.21 & 15.07 & 12.83 & 28.79 & 34.28 \\
\hline $14 X-1,33-35$ & 103.33 & 3.61 & 26.93 & 23.97 & 55.93 & 62.22 \\
\hline $15 X-1,31-33$ & 112.91 & 1.12 & 7.60 & 11.79 & 28.89 & 37.69 \\
\hline $16 \mathrm{X}-1,30-32$ & 122.50 & & 15.90 & 9.55 & 39.13 & 39.12 \\
\hline $17 X-1,31-33$ & 132.11 & & 11.49 & 13.33 & 26.52 & 38.97 \\
\hline $18 X-1,31-33$ & 141.81 & & 10.50 & 7.99 & 24.27 & 29.27 \\
\hline $19 \mathrm{X}-1,30-32$ & 151.40 & 0.68 & 6.16 & 5.68 & 15.21 & 16.67 \\
\hline $21 X-1,30-32$ & 170.80 & 1.51 & 7.75 & 9.35 & 17.24 & 22.90 \\
\hline $22 X-1,31-33$ & 180.41 & 0.62 & 0.00 & 2.52 & 11.34 & 10.32 \\
\hline $23 \mathrm{X}-1,30-32$ & 190.10 & 0.55 & 3.16 & 2.14 & 7.39 & 8.50 \\
\hline $24 \mathrm{X}-1,32-34$ & 199.72 & 0.74 & 7.21 & 6.12 & 15.63 & 23.84 \\
\hline $25 \mathrm{X}-1,32-34$ & 209.42 & 2.49 & 12.55 & 7.99 & 26.44 & 19.83 \\
\hline $26 \mathrm{X}-1,31-33$ & 219.01 & 5.66 & 5.46 & 10.86 & 27.02 & 31.05 \\
\hline $27 \mathrm{X}-1,31-33$ & 228.71 & 3.69 & 7.11 & 9.76 & 25.54 & 20.24 \\
\hline $28 \mathrm{X}-1.30-32$ & 238.30 & 1.78 & 1.01 & 4.34 & 11.71 & 15.50 \\
\hline $29 \mathrm{X}-1,31-33$ & 247.91 & & 7.29 & 5.71 & 15.97 & 17.99 \\
\hline $30 X-1,31-33$ & 257.61 & 3.92 & 6.64 & 8.13 & 19.78 & 25.04 \\
\hline $31 X-1,30-32$ & 267.30 & 3.00 & 8.78 & 7.07 & 21.50 & 29.42 \\
\hline $32 X-1.30-32$ & 277.00 & 2.04 & 10.0 & 7.78 & 24.07 & 28.46 \\
\hline $33 \times-1,30-32$ & 286.60 & 3.21 & 12,86 & 6.28 & 21.22 & 23.51 \\
\hline $34 \mathrm{X}-1,32-34$ & 296.32 & 2.70 & 9.12 & 9.75 & 28.17 & 39.52 \\
\hline $35 \times-1,31-33$ & 306.01 & 4.67 & 5.09 & 6.26 & 24.84 & 39.52 \\
\hline $36 \mathrm{X}-1,31-33$ & 315.61 & & 10.89 & 8.98 & 26.13 & 35.29 \\
\hline $37 \mathrm{X}-1,30-32$ & 325.30 & & 7.83 & 4.88 & 12.71 & 17.17 \\
\hline $38 \times-1,30-32$ & 334.60 & 3.45 & 7.65 & 5.04 & 16.36 & 17.56 \\
\hline $39 X-1,31-33$ & 344.21 & 2.63 & 12.08 & 7.27 & 28.61 & 40.58 \\
\hline $40 X-1,31-33$ & 353.91 & 1.03 & 7.72 & 8.45 & 30.18 & 29.99 \\
\hline $41 X-1,31-33$ & 363.41 & 2.37 & 10.72 & 11.94 & 30.10 & 40.03 \\
\hline $42 X-1,30-32$ & 373.00 & 3.44 & 10.47 & 9.79 & 24.22 & 26.71 \\
\hline $43 X-1,30-32$ & 382.70 & 0.03 & 6.05 & 4.25 & 15.66 & 14.07 \\
\hline $44 X-1,30-32$ & 392.30 & 4.40 & 14.91 & 10.47 & 29.00 & 36.09 \\
\hline $45 X-1.30-32$ & 402.00 & 1.51 & 16.05 & 5.46 & 23.80 & 24.52 \\
\hline $46 \mathrm{X}-1,30-32$ & 411.60 & 7.28 & 17.33 & 12.19 & 47.10 & 55.25 \\
\hline $47 X-1,30-32$ & 421.20 & 2.06 & 7.87 & 7.87 & 19.52 & 19.01 \\
\hline $48 \mathrm{X}-1,30-32$ & 430.90 & & 6.84 & 5.01 & 10.43 & 8.84 \\
\hline $49 X-1,30-32$ & 440.50 & 5.13 & 14.44 & 13.57 & 34.45 & 29.10 \\
\hline $50 X-1,30-32$ & 450.10 & 2.46 & 11.25 & 8.49 & 24.73 & 25.52 \\
\hline $51 X-1,30-32$ & 459.60 & 1.76 & 7.52 & 5.72 & 23.94 & 26.01 \\
\hline $52 X-1,30-32$ & 469.20 & 6.99 & 14.60 & 8.02 & 44.84 & 51.85 \\
\hline $53 \mathrm{X}-1,32-34$ & 478.92 & 8.89 & 22.16 & 7.15 & 51.47 & 49.35 \\
\hline $54 X-1,30-32$ & 488.50 & 1.26 & 10.26 & 6.52 & 24.22 & 27.97 \\
\hline $55 X-1,30-32$ & 498.10 & 1.23 & 9.13 & 7.58 & 29.49 & 28.86 \\
\hline $56 \mathrm{X}-1,30-32$ & 507.70 & 6.85 & 12.56 & 10.05 & 48.54 & 59.51 \\
\hline $57 X-1,30-32$ & 517.40 & 2.65 & 14.05 & 8.78 & 41.32 & 42.36 \\
\hline $58 \mathrm{X}-1,30-32$ & 527.00 & 9.09 & 14.20 & 17.49 & 63.21 & 77.97 \\
\hline $59 X-1,30-32$ & 536.70 & 0.55 & 12.26 & 5.82 & 28.26 & 24.47 \\
\hline & Average & 2.82 & 10.58 & 8.94 & 27.04 & 31.48 \\
\hline & $\mathrm{SD}$ & 2.21 & 5.29 & 4.31 & 11.91 & 14.01 \\
\hline
\end{tabular}

Note: $\mathrm{SD}=$ standard deviation, blank = no measurement. 